\title{
Higher Nutritional Status of Lung Cancer Cachexia Patients is Associated with Higher Functional Capacity and Appetite
}

Received, 15 November 2018 Accepted, 11 Desember 2018

Link to DOI:

10.25220/WNJ.V02.i2.0006

Journal Website:

www.worldnutrijournal.org

Diana Sunardi ${ }^{1}$, Saptawati Bardosono ${ }^{1}$

1. Department of Nutrition, Faculty of Medicine, Universitas Indonesia, Cipto Mangunkusumo Hospital

Abstract

Introduction Lung cancer is highly prevalent and is the major cause of cancer-related deaths worldwide. Severity of weight loss has been shown to have a negative impact on patients' performance and quality of life. Early detection of anorexia and decline of functional capacity will be very useful to prevent severe weight loss and for better prognosis. This study aims to find relationship between nutritional status with functional capacity and appetite in lung cancer cachexia patients

Methods A cross sectional study was conducted in Dharmais Cancer Hospital. Subjects were adult patients over 18 years old with newly diagnosed lung cancer or a minimal of 2 months post chemotherapy or radiotherapy, taken consecutively from inpatients and outpatients. Patients with hepatic cirrhosis and end stage kidney disease were excluded. Parameters of functional capacity (ECOG) and appetite (VAS and SNAQ) were the dependent variables, while nutritional status were the independent.

Results Subjects eligible for the study were 47 patients: $55.3 \%$ men and $44.7 \%$ women with the age range from 36-79 years old. There were significant relationship between body weight and BMI with ECOG score $(p=0.038 ; p=0.016)$. Body weight, BMI and weight loss related significantly with VAS appetite $(p=0.016 ; p=0.006 ; p=0.028)$ and SNAQ score $(p=0.005 ; p=0.009 ; p=0.028 ; p=0.014)$. These result indicate that subjects with a higher $\mathrm{BMI}$ and a lower weight loss have a better physical performance and appetite. There were significant relation between hemoglobin level, anemia status and serum albumin level with VAS appetite $(p=0.004 ; p=0.004 ; p=0031)$ and risk for anorexia $(p=0.038 ; p=0.004)$. This result indicate that subjects with normal hemoglobin and albumin have good appetite and lower risk for anorexia.

Conclusion higher nutritional status, including body weight, BMI, hemoglobin and albumin positively affect functional capacity and appetite.

Keywords lung cancer, albumin, hemoglobin, appetite, functional capacity

\section{Introduction}

The incidence of cancer is increasing worldwide and with it the prevalence of malnutrition, which may vary between 40 and $80 \%$. The etiology of

\footnotetext{
Corresponding author:

Diana Sunardi, MD

Faculty of Medicine - Universitas Indonesia

Cipto Mangunkusumo Hospital

Jl. Salemba Raya no. 6, Jakarta 104630

Email: dianasunardi@yahoo.com
}

malnutrition in cancer patients is complex, multifactorial and may be influenced by the location and type of tumor, stage of the disease, side effects of the treatment, socioeconomic status, functional performance, symptoms of nutritional impact, need for fasting and inadequate nutritional therapy, as well as medical staff awareness about the importance of nutritional status for the prognosis and quality of life of hospitalized patients. . $^{1,2}$ Weight loss is the primary defining feature of the wasting syndrome, cancer cachexia. ${ }^{3}$ Cachexia is a condition where skeletal muscle mass and adipose tissue are 
progressively wasted. Incidence of cancer cachexia in patients with a cancer is more than $50 \%$. The presence of some degree of weight loss in $60 \%$ of some gastrointestinal tumors and $80 \%$ of lung cancers upon diagnosis suggest that cancer cachexia should be considered as an "early event". Lung cancer is highly prevalent and the major cause of cancer-related deaths worldwide. Severity of weight loss has been shown to have a negative impact on patients' performance and quality of life. ${ }^{4,5}$

The prominent clinical feature of cachexia is weight loss, anorexia, inflammation, insulin resistance, and increased muscle protein breakdown. ${ }^{6}$ Patients with cancer cachexia frequently report reduced appetite and have reduced food intake. Paradoxically, it is still unclear what proportion of cancer related weight loss can be attributed to reduced dietary intake and how much cancer-related weight loss could be prevented or reversed by increasing nutritional intake. ${ }^{7}$ Normal appetite involves peripheral and central pathways. Peripheral appetite control has four phases of normal hunger and satiety cycles. The four phases are gastric motility phase, post absorptive phase, metabolic phase and ileal phase. Central appetite control depends on the balance between energy inputs and resting energy expenditure governed by a parallel system of NPY and pro-opiomelanocortin (POMC). NPY and POMC are regulated primarily by leptin and serotonin. NPY and agouti-gene related transcript (AGRP) is the main appetite stimulating central neurotransmitters with separate but synergistic appetite response. POMC reduces appetite by opposing the actions of NPY. Leptin is the dominant negative peripheral NPY regulator secreted by white adipocytes. Serotonin inhibits NPY neuron activity cause satiety. ${ }^{8}$

Cachexia contributes substantially to morbidity in cancer patients. It is associated with symptoms such as fatigue, weakness, poor physical performance, and thus leads to a lower self-rated quality of life. Patients who continue to lose weight while receiving palliative chemotherapy have reduced global quality of life and performance scores when compared to those whose weight loss stabilises. ${ }^{6}$

The assessment of the body weight change, nutritional status, and non-obvious symptoms that affect nutrition tend to be relatively low on the priority list. ${ }^{9}$ The identification of factors that can be assessed during the hospital admission can alert the medical and healthcare staff about the need for evaluation and early nutritional intervention to avoid further complications, an increase in length of hospital stay and costs. ${ }^{10}$

The above description shows that functional capacity and appetite are prognostic factors for cancer patients. Then lead us to questions, are there relation between nutritional status with functional capacity and appetite. The aim of the study was to find relation between nutritional status with functional capacity and appetite.

\section{Methods}

A cross sectional study was conducted in Dharmais Cancer Hospital, as part of a larger study on the association between cytokines pro and antiinflammatory. The study was approved by the Committee for Ethics in Research of the Dharmais Cancer Hospital. Patient recruitment took place from September 2017 to May 2018. Subjects were lung cancer patients newly diagnosed or a minimal of 2 months post chemotherapy or radiotherapy, taken consecutively from outward and inward. Sixty patients agree to take part of the study by signing written informed consent, 47 were eligible to take part in this study.

The anthropometric variables, body height and weight, was measured twice and then calculated the average by medical doctor with SECA portable stadiometer and SECA body weight scale with a variation of $0.01 \mathrm{~cm}$ and $0.1 \mathrm{~kg}$ precision. BMI was calculated as weight $(\mathrm{kg}) /$ height squared $(\mathrm{m} 2)$. The performance status was obtained from the scale developed by the Eastern Cooperative Oncology Group (ECOG), which ranks the functional capacity in five levels. Level zero indicates the individual is fully active, and level four indicates the bedridden and unable to perform self care activities. Appetite was measured with VAS, and the risk for anorexia was measured using SNAQ. Serum albumin and hemoglobin, obtained from blood vein analysis, were measured as parameters of nutritional status. All laboratory values were determined using routine automated analyzers at the Department of Clinical Pathology of Dharmais Cancer Hospital.

The database was structured using the Microsoft Excel 2013 software, and the analyses were done using the Statistical Package for Social Sciences (SPSS) software version 20 for Windows. 
The numeric variables were described with mean (standard deviation) for normal distributed data or median (minimal-maximal) value for otherwise. Comparison between patient groups was assessed using T-test or Mann-Whitney, according to data distribution. The Chi Squared or Mann-Whitney U test were utilized to associate clinical and biochemical parameters, and malnutrition. Statistical significance was determined as $p<0.05$ with a two-sided test.

\section{Results}

Sixty patients agreed to take part of the study, 47 of them fulfilled the study subject criteria. Subjects of the study composed of $55.3 \%$ men and $44.7 \%$ women, with the age range from 36-79 years old. The highest age range was between $51-65$ years old (40.4\%).

Table 1. Subject characteristics

\begin{tabular}{lcc}
\hline Characteristics & & Percentage (\%) \\
\hline Sex & & \\
$\quad$ male & 26 & 55.3 \\
$\quad$ female & 21 & 44.7 \\
Age, year & & \\
$\quad 36-50$ & 14 & 29.8 \\
$51-65$ & 19 & 40.4 \\
$>65$ & 14 & 29.8 \\
\hline
\end{tabular}

The median body weight was $49.2(35-78) \mathrm{kg}$, with average BMI $20.4 \pm 4.0 \mathrm{~kg} / \mathrm{m}^{2}$. The average weight loss in 6 months was $14.9 \pm 7.8$, while the median in 12 months was $14.7(0-32.6) \mathrm{kg}$. The average albumin level was $3.2 \pm 0,6 \mathrm{~g} / \mathrm{dL}$, hypoalbuminemia occurred in 48.9. The average hemoglobin level was $11.3 \pm 1.7 \mathrm{~g} / \mathrm{dL}$, anemia was found in $63.8 \%$.

There were significant relationship between body weight and ECOG score, VAS appetite and SNAQ score. Body mass index was significantly related to ECOG score. Weight loss in six months was related with SNAQ score, while weight loss in twelve months were related with VAS appetite and SNAQ score. There were significant relation between hemoglobin level and VAS appetite, and anemia status was related significantly with appetite status. This result indicates that subjects with normal hemoglobin have a good appetite. Anemia status was related significantly with anorexia risk status, which indicates that subjects with anemia have a higher risk for anorexia. We found that there were significant relation between albumin level and VAS appetite and SNAQ score (Table 2).

\section{Discussion}

Nutritional and functional deterioration are so frequently encountered in cancer patients that they are often accepted as part of the disease. The majority of patients with advanced lung cancer also present with malnourishment and, subsequently, with hypoalbuminemia and anemia. ${ }^{11}$ There were significant relationship between body weight and ECOG score, VAS appetite and SNAQ score. Body mass index (BMI) was significantly related to ECOG score. ECOG score is the picture of one functional capacity, this result shows us that body weight and BMI significantly affected functional capacity. A Greek prospective study conducted with lung cancer showed an association between the performance status and the weight loss of more than $5 \%$ in the last 3 months, percent weight loss is as an indicative of a considerable deterioration of the nutritional status. ${ }^{10}$

This study found that weight loss in six months was related with SNAQ score, while weight loss in twelve months was related with VAS appetite and SNAQ score. Nasrah et $\mathrm{al},{ }^{7}$ did not found a correlation between weight loss and appetite. Anorexia or loss of appetite was the symptom most closely linked to both reduced dietary intake and weight loss, as well as being an independent explanatory variable for weight change in multivariate analysis. This is consistent with the concept that the symptom of anorexia can promote weight loss through reduced food intake, but also that anorexia can be an indicator of cancer- related changes in central nervous system signaling that promote tissue loss independent of food intake. 
Table 2. Nutritional status related to ECOG score, VAS appetite and SNAQ score

\begin{tabular}{|c|c|c|c|c|c|c|c|c|c|}
\hline \multirow[t]{3}{*}{ Nutritional status } & \multicolumn{3}{|c|}{ ECOG score } & \multicolumn{3}{|c|}{ VAS appetite } & \multicolumn{3}{|c|}{ Risk of anorexia } \\
\hline & $\begin{array}{r}\text { Low } \\
\mathrm{N}=21\end{array}$ & $\begin{array}{l}\text { Good } \\
\mathrm{N}=26\end{array}$ & $\mathrm{p}$ & $\begin{array}{l}\text { Low } \\
n=15\end{array}$ & $\begin{array}{l}\text { Good } \\
n=32\end{array}$ & $\mathrm{p}$ & Risk & $\begin{array}{c}\text { Not at Risk } \\
n=21\end{array}$ & $\mathrm{p}$ \\
\hline & & & & & & & $\mathrm{n}=26$ & & \\
\hline Body weight, Kg & $48.9 \pm 9.1$ & $54.7 \pm 9.4$ & $p=0.038$ & $47.2 \pm 7.5$ & $54.4 \pm 9.8$ & $p=0.016$ & $48(35-75)$ & $55.8 \pm 9.4$ & $p=0.005$ \\
\hline Height, Kg & $161.6 \pm 8.0$ & $159.2 \pm 7.5$ & $\mathrm{p}=0.289$ & $162.2 \pm 9.2$ & $159.3 \pm 6.9$ & $\mathrm{p}=0.244$ & $160.8 \pm 8.1$ & $159.5 \pm 7.3$ & $\mathrm{p}=0.583$ \\
\hline BMI, $\mathrm{Kg} / \mathrm{m}^{2}$ & $18.9 \pm 3.8$ & $21.6 \pm 3.7$ & $p=0.016$ & $18.1 \pm 3.7$ & $21.5 \pm 3.7$ & $\mathrm{p}=0.006$ & $\begin{array}{c}18.5(13.1- \\
27.1)\end{array}$ & $22.0 \pm 3.7$ & $\mathrm{p}=0.009$ \\
\hline \multicolumn{10}{|l|}{ Weight loss: } \\
\hline 6 months & $14.9 \pm 8.3$ & $\begin{array}{l}12.6(5,1- \\
32.6)^{*}\end{array}$ & $\mathrm{p}=0.923$ & $18.0 \pm 8.0$ & $11.8(0-32.0)$ & $\mathrm{p}=0.055$ & $17.2 \pm 7.7$ & $12.2 \pm 7.1$ & $p=\mathbf{0 . 0 2 8}$ \\
\hline 12 months & $16.7 \pm 9.4$ & $15.3 \pm 7.6$ & $\mathrm{p}=0.588$ & $19.8 \pm 8.8$ & $14.1 \pm 7.7$ & $p=0.028$ & $18.6 \pm 8.3$ & $12.7 \pm 7.5$ & $p=0.014$ \\
\hline Hemoglobin, mg/dL & $10.8 \pm 1.7$ & $11.7 \pm 1.7$ & $\mathrm{p}=0.070$ & $10.2 \pm 1.5$ & $11.8 \pm 1.6$ & $P=0.004$ & $10.9 \pm 1.7$ & $11.8 \pm 1.7$ & $\mathrm{p}=0.070$ \\
\hline Anemia & 15 & 15 & $\mathrm{p}=0.330$ & 14 & 16 & $\mathrm{P}=\mathbf{0 . 0 0 4}$ & 20 & 10 & $p=\mathbf{0 . 0 3 8}$ \\
\hline Normal & 6 & 11 & & 1 & 16 & & 6 & 11 & \\
\hline Albumin, g/dL & $3.0 \pm 0.5$ & $3.3 \pm 0.6$ & $\mathrm{p}=0.089$ & $2.9 \pm 0.5$ & $3.3 \pm 0.6$ & $P=0.031$ & $3.0 \pm 0.5$ & $3.4 \pm 0.6$ & $p=0.004$ \\
\hline Hypoalbuminemia & 12 & 11 & $\mathrm{p}=0.312$ & 10 & 13 & $\mathrm{P}=0.096$ & 16 & 7 & $\mathrm{p}=0.054$ \\
\hline Normal & 9 & 15 & & 5 & 19 & & 10 & 14 & \\
\hline
\end{tabular}


Studies in cancer-bearing rodents have shown elevated levels of inflammatory cytokines in the hypothalamus which can induce anorexia. The anorexia induced by hypothalamic inflammatory mediators can be reversed by melanocortin type- 4 receptor blockade, but centrally acting cytokines such as IL-1b can also trigger muscle wasting, independent of central melanocortin signaling. We hypothesize that for some patients with cancer cachexia and anorexia, the presence of anorexia is a marker for cancer-related hypothalamic inflammatory cytokine signaling. If this central signaling also directly triggers tissue catabolism and weight loss in humans, its effects would likely not be reversed by increased nutritional intake alone. This in turn would explain the independent explanatory power of anorexia for weight change, even when dietary intake is taken into account. ${ }^{12,13,14}$

There was a significant relation between hemoglobin level and VAS appetite, and anemia status was related significantly with Appetite status. This result indicate that subjects with normal hemoglobin have a good appetite. Anemia status was related significantly with anorexia risk status, this result indicate that subjects with anemia have a higher risk for anorexia. Kalantar Zadeh et al, on their study in hemodialysis patients found a similar trend was also found for blood hemoglobin, patients with a diminished appetite had a slightly lower hemoglobin concentration. ${ }^{15}$ This result shows us that to maintain good appetite we must maintain our cancer patient's hemoglobin. We found that there was a significant relation between Albumin level and VAS appetite and SNAQ score (Table 1). Arrieta et all, ${ }^{11}$ found the same result: hypoalbuminemia was associated with loss of appetite in cancer patients. A need for normal albumin level, by this result, is needed for a good appetite.

Our results in this study shows that maintaining body weight is very important for cancer patients in order to have a good physical performance, which will also affect their prognosis. This study give an evidence that cancer patients appetite affected by many factors. Those factors were body weight, BMI, weight loss, hemoglobin and albumin level.

In conclusion, this study shows that nutritional status, including body weight, BMI, hemoglobin and albumin are related with functional capacity and appetite. Maintaining body weight,
BMI, normal hemoglobin and albumin are keys factors of functional capacity and appetite. Serum Albumin and hemoglobin are simple and cheap tests, clinical markers that may be used to assess the risk of anorexia in cancer patients objectively. Screening for nutritional status, including body weight, BMI, history of weight loss and biochemical markers are very important to do in cancer patients.

\section{Conflict of Interest}

None of the other authors have conflict of interest. No educational grant is provided to the rest of authors.

\section{Open Access}

This article is distributed under the terms of the Creative Commons Attribution 4.0 International Licence(http://creativecommons.org/licenses/by/4.0 ), which permits unrestricted use, distribution, and reproduction in any medium, provided you give appropriate credit to the original author(s) and the source, provide a link to the Creative Commons license, and indicate if changes were made.

\section{Acknowledgement}

We would like to thank all the participants of this study, we also thank the Management, the Doctors, Paramedics, and Nutritionist of the Dharmais Cancer Hospital for their support.

\section{References}

1. Pressoir M, Desné S, Berchery D, et al. Prevalence, risk factors and clinical implications of malnutrition in french comprehensive cancer centres. Br J Cancer 2010;102(6):966-971. [Google Scholar]

2. Wu BW, Yin T, Cao WX, et al. Clinical application of subjective global assessment in Chinese patients with gastrointestinal cancer. World J Gastroenterol 2009;15(28):3542-3549. [Google Scholar]

3. Fearon K, Strasser F, Anker SD, et al. Definition and classification of cancer cachexia: An international consensus. Lancet Oncol 2011;12(5):489-495. [Google Scholar] 
4. Inui A. Cancer anorexia-cachexia syndrome: current issues in research and management. CA Cancer J Clin 2002;52(2):72-91. [Google Scholar]

5. Topkan E, Yavuz AA, Ozyilkan O. Cancer cachexia: Pathophysiologic aspects and treatment options. Asian Pacific J Cancer Prev 2007;8(3):445-451. [Google Scholar]

6. Donohoe CL, Ryan AM, Reynolds JV. . Cancer cachexia: mechanisms and clinical implications. Gastroenterol Res Pract. 2011; 2011. [Google Scholar]

7. Nasrah R, Kanbalian M, Borch, C. Van Der , Swinton N, Wing S, Jagoe RT. Defining the role of dietary intake in determining weight change in patients with cancer cachexia. Clinical Nutrition 2018 Feb;37(1):235-41. [Google Scholar]

8. Davis MP, Dreicer R, Walsh D, Lagman R, LeGrand SB. Appetite and cancer-associated anorexia: A review. J Clin Oncol 2004;22(8):1510-1517. [Google Scholar]

9. Mariani L, Vullo, S. Lo, Bozzetti F. Weight loss in cancer patients: A plea for a better awareness of the issue. Support Care Cancer 2012;20(2):301-309. [Google Scholar]

10. Silva FR de M, Oliveira, M.G.O.A. de , Souza ASR, Figueroa JN, Santos CS. Factors associated with malnutrition in hospitalized cancer patients: a croos-sectional study. Nutr J 2015;14(1):123. [Google Scholar]

11. Arrieta O, Rm MO, Villanueva-Rodríguez $\mathrm{G}$, et al. Association of nutritional status and serum albumin levels with development of toxicity in patients with advanced non-small cell lung cancer treated with paclitaxelcisplatin chemotherapy: A prospective study. BMC Cancer 2010;10(22):1-7. [Google Scholar]

12. Ropelle ER, Pauli JR, Zecchin KG, et al. A central role for neuronal adenosine 5'monophosphate-activated protein kinase in cancer-induced anorexia. Endocrinology 2007;148(11):5220-5229. [Google Scholar]

13. Grossberg AJ, Scarlett JM, Marks DL. Hypothalamic mechanisms in cachexia. Physiol Behav 2010;100(5):478-489. [Google Scholar]

14. Braun TP, Zhu X, Szumowski M, et al. Central nervous system inflammation induces muscle atrophy via activation of the hypothalamic-pituitary-adrenal axis. J Exp Med 2011;208(12):2449-2463. [Google Scholar]

15. Kalantar-Zadeh K, Block G, McAllister CJ, Humphreys MH, Kopple JD. Appetite and inflammation, nutrition, anemia, and clinical outcome in hemodialysis patients. Am J Clin Nutr 2004;80(2):299-307. [Google Scholar] 